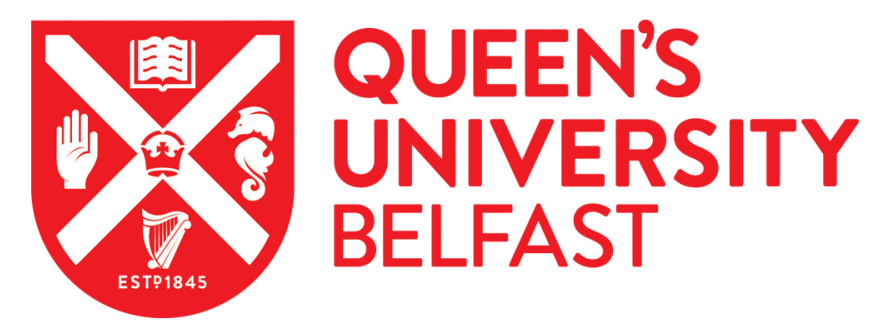

\title{
Quantifying Aflatoxin B1 in peanut oil using fabricating fluorescence probes based on upconversion nanoparticles.
}

Sun, C., Li, H., Koidis, A., \& Chen, Q. (2016). Quantifying Aflatoxin B1 in peanut oil using fabricating fluorescence probes based on upconversion nanoparticles. SPECTROCHIMICA ACTA PART A-MOLECULAR AND BIOMOLECULAR SPECTROSCOPY, 165, 120-126. https://doi.org/10.1016/j.saa.2016.04.040

Published in:

SPECTROCHIMICA ACTA PART A-MOLECULAR AND BIOMOLECULAR SPECTROSCOPY

Document Version:

Peer reviewed version

Queen's University Belfast - Research Portal:

Link to publication record in Queen's University Belfast Research Portal

Publisher rights

Copyright 2016 Elsevier.

This manuscript is made available under a Creative Commons Attribution-NonCommercial-NoDerivs License

(https://creativecommons.org/licenses/by-nc-nd/4.0/), which permits distribution and reproduction for non-commercial purposes, provided the author and source are cited.

\section{General rights}

Copyright for the publications made accessible via the Queen's University Belfast Research Portal is retained by the author(s) and / or other copyright owners and it is a condition of accessing these publications that users recognise and abide by the legal requirements associated with these rights.

Take down policy

The Research Portal is Queen's institutional repository that provides access to Queen's research output. Every effort has been made to ensure that content in the Research Portal does not infringe any person's rights, or applicable UK laws. If you discover content in the

Research Portal that you believe breaches copyright or violates any law, please contact openaccess@qub.ac.uk. 
1 Quantifying Aflatoxin $\mathrm{B}_{1}$ in peanut oil using fabricating fluorescence probes

2 based on upconversion nanoparticles

4 Cuicui Sun, Huanhuan Li, Anastasios Koidis, Quansheng Chen*

5 School of Food and Biological engineering, Jiangsu University, Zhenjiang, 212013, P. R. China.

6 Institute for Global Food Security, Queen's University Belfast, BT95GN, Northern Ireland, United

$7 \quad$ Kingdom. 


\section{ABSTRACT}

10 Rare earth doped upconversion nanoparticles convert near-infrared excitation light into visible

11 emission light. Compared to organic fluorophores and semiconducting nanoparticles, upconversion

12 nanoparticles (UCNPs) offer high photochemical stability, sharp emission bandwidths, and large

13 anti-Stokes shifts. Along with the significant light penetration depth and the absence of

14 autofluorescence in biological samples under infrared excitation, these UCNPs have attracted more

15 and more attention on toxin detection and biological labelling. Herein, the fluorescence probe based

16 on UCNPs was developed for quantifying Aflatoxin $B_{1}\left(\mathrm{AFB}_{1}\right)$ in peanut oil. Based on a specific

17 immunity format, the detection limit for $\mathrm{AFB}_{1}$ under optimal conditions was obtained as low as 0.2

$18 \mathrm{ng} \cdot \mathrm{mL}^{-1}$, and in the effective detection range 0.2 to $100 \mathrm{ng} \cdot \mathrm{mL}^{-1}$, good relationship between

19 fluorescence intensity and $\mathrm{AFB}_{1}$ concentration was achieved under the linear ratios up to 0.90.

20 Moreover, to check the feasibility of these probes on $\mathrm{AFB}_{1}$ measurements in peanut oil, recovery

21 tests have been carried out.A good accuracy rating (\%) was obtained in this study. results showed

22 that the nanoparticles can be successfully applied for sensing AFB 1 in daily edible oils.

23 Keywords: rapid toxin detection; biological labelling; upconversion nanoparticles; Fluorescence

24 probes 
China and India regions is the world's biggest market for peanut and its derivatives (in particular peanut oil) in terms of productions well as consumption (Sanders Iii et al., 2014). Several survey studies have shown that mold will grow quickly, and the possible presence of aflatoxins would transfer into peanut oil, when peanuts are stored for weeks in humid conditions (Klu \& Chen, 2015).

Aflatoxins are a group of highly toxic secondary metabolites produced mainly by Aspergillus flavus and Aspergillus parasiticus on a variety of food products (K. Chen et al., 2014). These toxins are known to be potent carcinogens, teratogens, mutagens, and immunosuppression and pose harmful threat to animal and human health (Xia et al., 2013). Naturally occurring aflatoxins are composed of $\mathrm{B}_{1}, \mathrm{~B}_{2}, \mathrm{G}_{1}$ and $\mathrm{G}_{2}$ types. Among them, aflatoxin $\mathrm{B}_{1}\left(\mathrm{AFB}_{1}\right)$ is the most abundant and carcinogenic (Passone, Girardi, \& Etcheverry, 2013). Since peanut oil is widely consumed as diet in the Asian region, even low levels of contamination may cause severe health and safety incidents (Luongo et al., 2013; Quiles, Manyes, Luciano, Mañes, \& Meca, 2015; Van de Perre, Jacxsens, Lachat, El Tahan, \& De Meulenaer, 2015). Therefore, determination of $\mathrm{AFB}_{1}$ in peanut and its derivatives becomes a subject of great importance for industries and regulators alike.

A wide range of methods are currently available, including thin layer chromatography (TLC), spectrometry (Busman, Liu, Zhong, Bobell, \& Maragos, 2014), gas chromatography(Ceker, Agar, Alpsoy, Nardemir, \& Kizil, 2014), High-performance liquid chromatography (HPLC)(Herzallah, 2009), fluorescence polarization assays(Maragos, 2009), radio immunoassays(Waliyar, Reddy, \& Lava-Kumar, 2009), enzyme-linked immunosorbent assay(Sai et al., 2010) (ELISA) and fiberoptic based immunoassays(Kozlov et al., 2004), which have been used for the detection of aflatoxins. However, most of these techniques require well equipped laboratories, trained personnel, harmful 
solvents, and are time-consuming. Therefore, the demand for developing a rapid and sensitive method for sensing aflatoxins is urgent.

In optical detection methods, conventional downconversion fluorescent materials, such as semiconductor nanoparticles, dye-coupled hybrid materials and mesoporous silica, are fluorophores that are commonly used in biological studies and clinical application because of their unique features (Mnoyan, Kirakosyan, Kim, Jang, \&Jeon, 2015); Sharma, Rawat, Solanki, \& Bohidar, 2015). One important intrinsic limitation, however, is that these materials usually emit one lower-energy photon after absorption of a higher-energy ultraviolet or visible photon. This lead to significant disadvantages, such as low light-penetration depth, potentially severe photodamage to living organisms (Sozer \& Kokini, 2014), and the autofluorescence (noise) of some biological samples. To solve these problems, the development of alternative biological luminescent labels through the use of up-converting rare-earth nanophosphors (UCNPs) has attracted a tremendous amount of attention due to the unique luminescence properties of rare-earth nanoparticles. Lanthanide-doped, near-infrared (NIR)-to-visible upconversion nanophosphors are capable of emitting strong visible fluorescence under the excitation of NIR light (typically $980 \mathrm{~nm}$ ). They have been shown to have significant advantages as fluorescent bio-label (Boyer, Manseau, Murray, \& van Veggel, 2010; Chatterjee, Rufaihah, \& Zhang, 2008; Fang et al., 2014; Huang, Yu, \& Chu, 2015; Ma, Liu, Han, Yang, \& Liu, 2015; Tian et al., 2015) over the traditional organic fluorophores due to their attractive optical and chemical features, including low toxicity (Chatterjee, Gnanasammandhan, \& Zhang, 2010; Zhang, Wu, Tang, Su, \& Lv, 2014), large stokes shifts (Ahn et al., 2016), high resistance to photobleaching(Feng Wang et al., 2011), blinking, photochemical stability (H. Q. Chen, Yuan, \& Wang, 2013) and the lack of both auto-fluorescence (Aramburu et al., 2015) and light scattering 
background (Zhou, Liu, \& Li, 2012). As a result, the signal-to-background ratio and sensitivity of the

71 detection can be greatly improved. Moreover, upconversion nanoparticles have also attracted

72 increasing interest due to their optical properties which can be achieved by adding a $\lambda$ exc. $=980 \mathrm{~nm}$ optical source used in fluorescence measurement. From the mentioned advantages above, we can conclude that the upconversion nanophosphors as color label has a high potential on the detection of toxin. the fields of biotechnology and contaminat detection, magnetic nanoparticles (MNPs) have been receiving considerable attention. Due to their magnetic properties, low toxicity, and biocompatibility, MNPs are useful for the separation of target antibiotics from a mixture of antibiotics and matrix substances. Additionally, MNPs help to concentrate the separated antibiotics into a small volume, which is suitable for impedance measurements(Z. Wang et al., 2013). Artificial antigen-modified MNPs were employed as immune sensing probes, and antibody functionalized UCNPs were used as rare earth doped upconversion nanoparticles and immunoproteins. Fig. 1 presents the scheme of this proposed fluorescence bioassay platform. Specific procedures are outlined as follows. Upconversion nanoparticles (UCNPs) were synthesized and functionalized. (2) The resultant water-soluble UCNPs were conjugated with anti-AFB 1 antibodies to produce biological fluorescent probes. (3) A fluorescence standard curve was prepared with different concentrations of AFB 1 (4)

91 Independent food samples were tested. As an efficient, specific, and technically simple biological 
92 probe, these selective sensors can be used for rapidly detecting toxin in food.

93

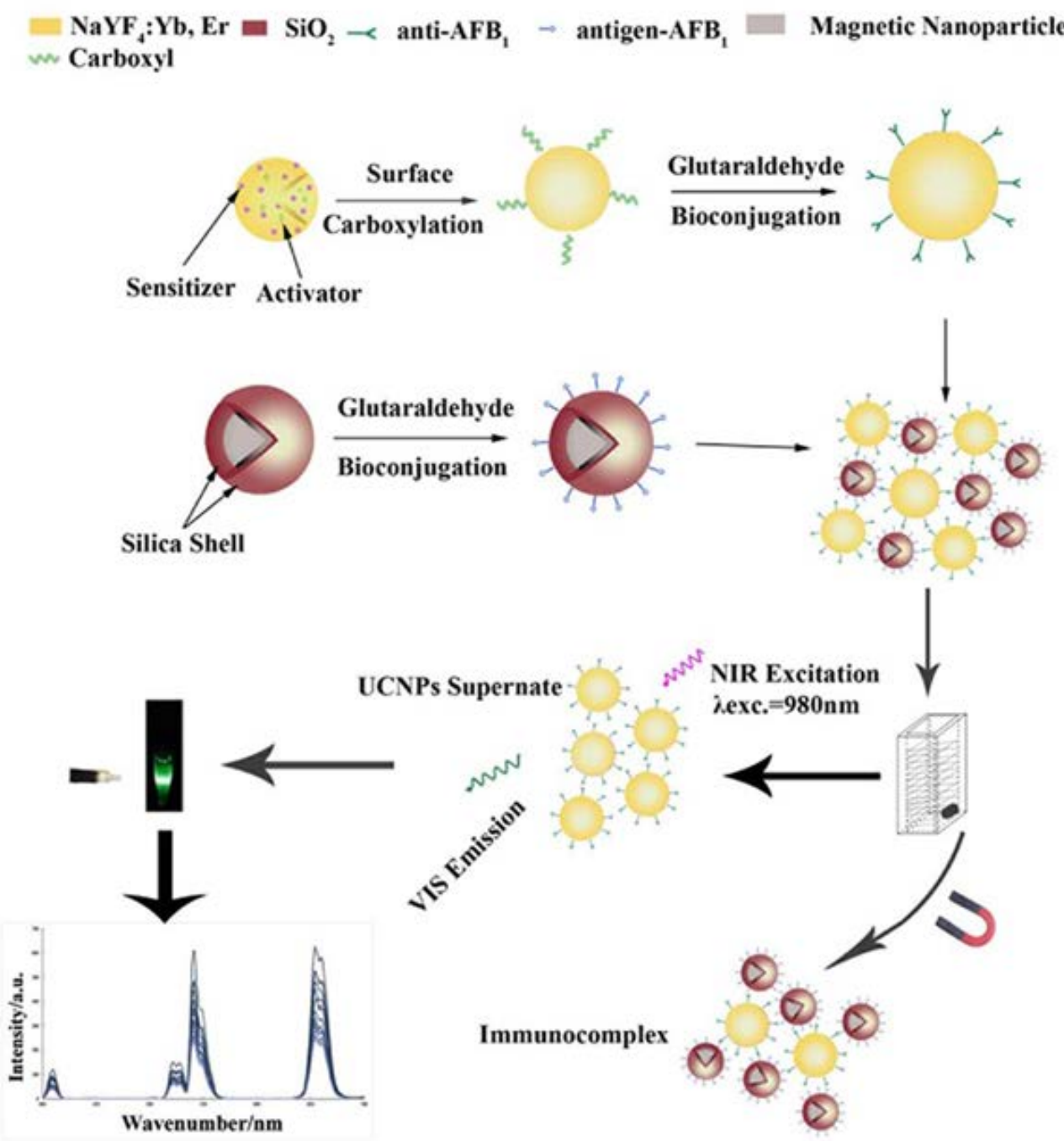

Fig. 1. Scheme of this proposed fluorescence bioassay platform.

\section{Materials and methods}

\subsection{Instruments}

The size and morphology of nanoparticles were determined using a JEM-2100HR transmission electron microscope (TEM, JEOL Ltd., Japan). X-ray diffraction (XRD) measurements were performed using a D8-advance instrument (Bruker AXS Ltd., Germany). Upconversion fluorescence 
spectra were measured using an F-7200 fluorescence spectrophotometer (Hitachi Co., U.S.A.) modified with an external 980nm laser (Beijing Hi-Tech Optoelectronic Co., China) instead of the internal excitation source. Fourier transform infrared spectrophotometer (FT-IR) spectra of the nanoparticles were obtained with a Nicolet Nexus 470 (Thermo Electron Co., U.S.A.) using a KBr detector.

\subsection{Reagents}

$\mathrm{AFB}_{1}$ standard solution, $\left(8 \mathrm{mg} \cdot \mathrm{mL}^{-1}\right.$ solution in methanol and working dilution by deionized water), $\mathrm{AFB}_{1}$-BSA antigen (extent of labeling 8-12 mol Aflatoxin $\mathrm{B}_{1}$ per mol BSA), monoclonal anti-AFB 1 antibody, (6 $\mathrm{mg} \cdot \mathrm{mL}^{-1}$ solution and working dilution by phosphate buffer solution) was obtained from Beijing Mozhidong Bio-tech (city. Country). Hydrated rare earth nitrate $\left(\mathrm{RECl}_{3} \cdot \mathrm{xH}_{2} \mathrm{O}\right.$, RE Y, Yb, Er, $\geq 99.99 \%)$, oleic acid $(\geq 90 \%)$ and octadecanoic acid ( $\geq 90 \%)$ were purchased from Sigma-Aldrich (Shanghai, China). In addition, $\mathrm{FeCl}_{3} \cdot 6 \mathrm{H}_{2} \mathrm{O}$, sodium fluoride, sodium hydroxide, methyl alcohol, toluene, ethyl alcohol, sodium citrate, 1,6-hexanediamine, anhydrous sodium acetate, glycol, bovine serum albumin (BSA, 96-99\%),25\% glutaraldehyde, tetraethyl orthosilicate (TEOS $\geq$ 98\%), and 3-aminopropyltrimethoxysilane (APTES) was all purchased from Sinopharm Chemical Reagent Co., Ltd. (Shanghai, China). All the chemicals used were of analytical grade. The water used was deionized.

\subsection{Synthesis and surface modification of rare-earth-doped}

Oleic acid-capped $\mathrm{NaYF}_{4}$ : Yb, Er UCNPs were synthesized according to the method reported in predecessors' research (F. Wang et al., 2010) with a few modifications. In a typical experiment, $2 \mathrm{ml}$ of $\mathrm{RECl}_{3}(0.2 \mathrm{M}, \mathrm{RE}=\mathrm{Y}(78 \%), \mathrm{Yb}(20 \%)$, Er (2\%)) in methanol were added to a $50 \mathrm{ml}$ flask containing $3 \mathrm{ml}$ oleic acid and $7 \mathrm{ml}$ 1-octadecene, and the solution was heated to $160{ }^{\circ} \mathrm{C}$ for $30 \mathrm{~min}$ 
and then cooled down to room temperature. Thereafter, $5 \mathrm{ml}$ methanol solution of $\mathrm{NH}_{4} \mathrm{~F}(1.6 \mathrm{mmol})$ and $\mathrm{NaOH}(1 \mathrm{mmol})$ was added and the solution was stirred for 30 min. After methanol evaporated, the solution was heated to $300{ }^{\circ} \mathrm{C}$ under argon for $1.5 \mathrm{~h}$ and cooled down to room temperature. The resulting nanoparticles were precipitated by the addition of ethanol, collected by centrifugation, washed with methanol and ethanol several times, and finally dried in an oven at $60{ }^{\circ} \mathrm{C}$.

The obtained oleic acid-capped UCNPs can disperse well in nonpolar solvents. However, for biological applications, hydrophobic UCNPs should be converted into hydrophilic UCNPs so as to be compatible with biomolecules, such as antibodies. Thus, surface modification of the hydrophobic UCNPs was performed via a ligand exchange process as described in predecessors' research (Ong, Ang, Alonso, \& Zhang, 2014). Briefly, a mixture of $2 \mathrm{mmol}$ sodium citrate in $15 \mathrm{ml}$ of diethylene glycol was first heated to $110{ }^{\circ} \mathrm{C}$ under argon for $30 \mathrm{~min}$. Oleic acid-capped UCNPs (10 mg) dispersed in cyclohexane and toluene were then added into the mixture and the reaction was heated to $160{ }^{\circ} \mathrm{C}$ for evaporation of cyclohexane and toluene. After complete evaporation, the reaction was further maintained at $160{ }^{\circ} \mathrm{C}$ for $3 \mathrm{~h}$. Water-soluble UCNPs were then collected by centrifugation, washed with ethanol and ultrapure water several times, and finally dispersed in ultrapure water.

\subsection{Preparation of amine-functionalized $\mathrm{Fe}_{3} \mathrm{O}_{4}$ magnetic nanoparticles}

Amine-functionalized $\mathrm{Fe}_{3} \mathrm{O}_{4}$ MNPs were prepared according to Gao's work (Gao, Gu, \& Xu, 2009). Briefly, a solution of $6.5 \mathrm{~g}$ 1,6-hexanediamine, $2.0 \mathrm{~g}$ anhydrous sodium acetate and $1.0 \mathrm{~g}$ $\mathrm{FeCl}_{3} 6 \mathrm{H}_{2} \mathrm{O}$ as a ferric source in $30 \mathrm{~mL}$ glycol was stirred vigorously at $50{ }^{\circ} \mathrm{C}$ to give a transparent solution. This solution was then transferred into a Teflon-lined autoclave and reacted at $198{ }^{\circ} \mathrm{C}$ for 6 h. The MNPs were then rinsed with water and ethanol (2 or 3 times) to effectively remove the 
solvent and unbound 1,6-hexanediamine, and then dried at $50{ }^{\circ} \mathrm{C}$ before characterization and application. During each rinsing step, the nanoparticles were separated from the supernatant by using magnetic force.

\subsection{Preparation of immunosensing probes and signal probes}

The artificial antigen conjugated MNPs and antibody conjugated immunosensing probes were fabricated with the classical glutaraldehyde method. Typically, $10 \mathrm{mg}$ of MNPs were dispersed in 5 $\mathrm{mL}$ of $10 \mathrm{mmol} / \mathrm{L}$ phosphate buffer solution ( $\mathrm{pH} 7.4$ ) by ultrasonication for $20 \mathrm{~min} .1 .25 \mathrm{~mL}$ of $25 \%$ glutaraldehyde was then added to the mixture. The mixture was shaken slowly at room temperature for $1 \mathrm{~h}$, and the $\mathrm{Fe}_{3} \mathrm{O}_{4}$ MNPs were separated by an external magnetic field and washed with PBS three times to remove the physically adsorbed glutaraldehyde. Subsequently, $11.67 \mu \mathrm{L}$ of $\mathrm{AFB}_{1}$-BSA antigen, at a concentration of $6 \mathrm{mg} \mathrm{mL}{ }^{-1}$, was added into $5 \mathrm{~mL}$ of a suspension of $\mathrm{Fe}_{3} \mathrm{O}_{4} \mathrm{MNPs}$ in PBS. The mixture was shaken slowly for $6 \mathrm{~h}$ at room temperature. The surplus biomolecules were removed by magnetic separation of the particles from the solution. The $\mathrm{AFB}_{1}-\mathrm{BSA}$ antigen conjugated MNPs were treated with $5 \mathrm{~mL}$ BSA at 3\% concentration in $10 \mathrm{mmol} / \mathrm{L}$ PBS at room temperature for $6 \mathrm{~h}$ to block the unreacted and nonspecific sites. Finally, the as-prepared probes were stored in $5 \mathrm{~mL}$ of $10 \mathrm{mmol} / \mathrm{L}$ PBS at $4{ }^{\circ} \mathrm{C}$ prior to use. The biofunctionalization of amino-modified, water-soluble UCNPs conjugated with monoclonal antibody, namely the preparation of the signal probes, was similar to that of the antigen conjugated MNPs described above. The prepared antigen conjugated $\mathrm{Fe}_{3} \mathrm{O}_{4}$ MNPs and antibody functionalized UCNPs were characterized by FT-IR spectroscopy.

\subsection{Sample preparation and measurement}

Twelve naturally contaminated peanut oil samples obtained from local supermarkets were 
treated according to official methods of China $(G B / T, 2003)$ with some modifications. Briefly, five

170 grams of each peanut oil sample and $5 \mathrm{~g} \mathrm{NaCl}$ were introduced into a $100 \mathrm{~mL}$ flask, and the 171 extracting solution (methanol:water; 7:3 (v:v)) was filled to the mark, completely mixed with the 172 compound and then the mixture was transferred into the cup of a homogenizer. The mixture was then 173 stirred at high-speed and extracted for $2 \mathrm{~min}$. Next, the resulting solution was filtered, and $10 \mathrm{~mL}$ of 174 filtrate was transferred into a $50 \mathrm{~mL}$ flask; water was filled to the mark, and the flask contents were 175 mixed to homogeneity. The resulting mixture was further filtered with glass fiber filter paper until 176 the filtrate was clear. For the standard addition and recovery experiments, the $\mathrm{AFB}_{1}$ standard 177 solutions were added to the peanut oil samples before adding the extracting solution. After the 178 complete chemical reaction and magnetism separation, fluorescence spectra of the obtained 179 supernatants (from 400 to $700 \mathrm{~nm}$ ) were measured with a fluorescence spectrophotometer equipped with a $980 \mathrm{~nm}$ laser excitation under the excitation power (1.3 W). Here, the $541 \mathrm{~nm}$ peak intensity emission wavelength was used.

\section{Results and Discussion}

\subsection{Characterization of the prepared upconversion nanoparticles and magnetic nanoparticles}

Results showed that toxin-specific antibodies with high selectivity and sensitivity were successfully conjugated onto the surface of UCNPs to yield UCNP-antibody probes, as illustrated in

Fig.1. Prior to the conjugation, the precursor UCNPs were first characterized by transmission electron microscopy (TEM), X-ray diffraction (XRD) and fluorescence spectral measurements, as shown in Fig 2. Successful surface modification, selectivities, sizes, and luminance and spectral properties of UCNPs before and after surface modification were validated by TEM and fluorescence 
UCNP structures and revealed that the particles were uniform with an average diameter of approximately $50 \mathrm{~nm}$ before and after surface modification and bioconjugation. The fluorescence spectra of the UCNPs showed the expected characteristic emission peaks at approximately 407, 542, and $657 \mathrm{~nm}$ upon NIR (980 nm) excitation, corresponding to blue, green, and red light, respectively (the naked-eye images in the inset show the visible intensity of the UCNPs). The peaks are ascribed to the transitions from the ${ }^{2} \mathrm{H}_{9 / 2},{ }^{4} \mathrm{~S}_{3 / 2}$, and ${ }^{4} \mathrm{~F}_{9 / 2}$ levels to the ${ }^{4} \mathrm{I}_{15 / 2}$ ground state of the $\mathrm{Er}^{3+}$ ion (L. Wang, Li, \& Li, 2007; Leyu Wang \& Li, 2006). The fluorescence properties were also retained, as both the oleic acid-capped UCNPs and the water-soluble UCNPs showed the same characteristic emission peaks upon NIR excitation. Additionally, the diffraction peaks of the XRD pattern in Fig. 2 (d) were identified as pure hexagonal $\beta$-phase $\mathrm{NaYF}_{4}$ crystals (JCPDS Standard Card No. 16-0334); no diffraction peaks corresponding to cubic phase crystals or other impurities were observed.

$\mathrm{a}$
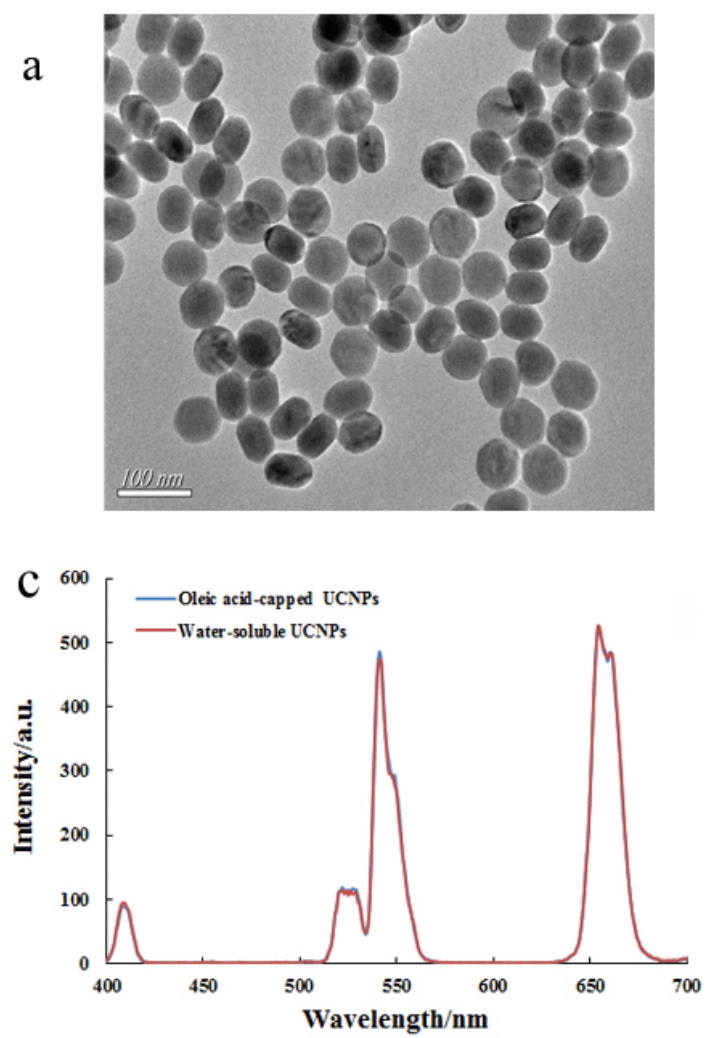

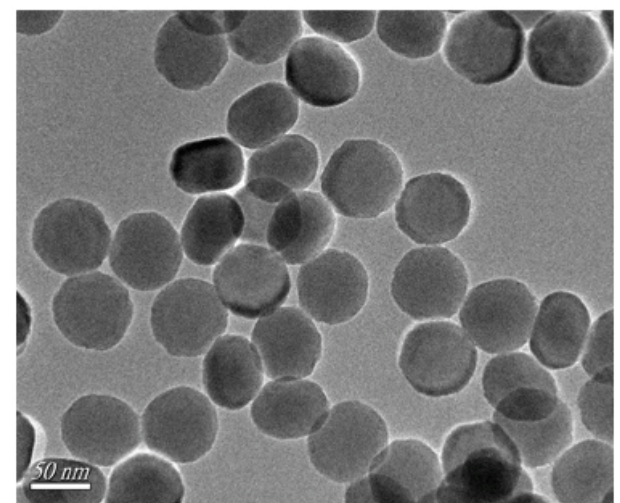

b

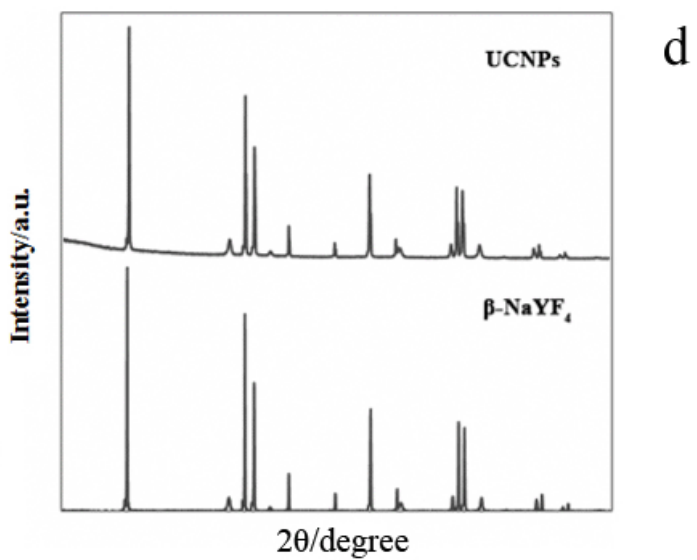

Fig. 2. TEM images of oleic acid-capped UCNPs (a) and water-soluble UCNPs (b), Fluorescence properties of 
The UCNPs used in this work were $\mathrm{Yb}$, Er ion-pair doped hexagonal phase $\mathrm{NaYF}_{4}$ nanoparticles. The hexagonal phase $\mathrm{NaYF}_{4}$ was reported to be one of the most efficient hosts for performing infrared to visible photon conversion when activated by $\mathrm{Yb}$, Er ion-pairs. During the experiment, we found that the reaction time and temperature were the two main influential factors in 210 the phase transition of $\mathrm{NaYF}_{4}$ UCNPs. In order to obtain hexagonal phase $\mathrm{NaYF}_{4}$, the reaction was 211 maintained at $300{ }^{\circ} \mathrm{C}$ for $1.5 \mathrm{~h}$.

212 The XRD pattern of $\mathrm{NaYF}_{4}$ : Yb, Er phosphor gives several reflections shown in Fig.2 (d) indicates 213 that the microballoon sphere are well-crystallized. In $\mathrm{Yb}^{3+}$ and $\mathrm{Er}^{3+}$ co-doped systems, $\mathrm{Yb}^{3+}$ ions act 214 as sensitizers and $\mathrm{Er}^{3+}$ ions as activators. The Debye Scherrer formula was used to calculate the 215 crystallite size of the synthesized phosphor and is given by $d=\frac{0.89 \lambda}{\beta \cos \theta}$, where $d$ is the crystallite size, $216 \lambda$ is the wavelength of the X-rays, $\beta$ is full width at half maximum and $\theta$ is the diffraction angle. The 217 average value of the crystallite size was found to be around $50 \mathrm{~nm}$ that confirms the formation of 218 nanostructured crystallites.

Fig. 3 (a, b) displays the TEM and selected area electron diffraction (SAED) images of 220 amino-modified MNPs confirming good dispersibility and morphology with an average size of about $22120 \mathrm{~nm}$. In addition, the crystalline structure and phase purity was determined by powder XRD as 222 shown in Fig. 3 (c). The positions and relative intensities of all diffraction peaks matched well with 223 those from the JCPDS card (No.52-0102) for magnetite. The sharp, strong peaks confirmed the 224 products were well crystallized. 

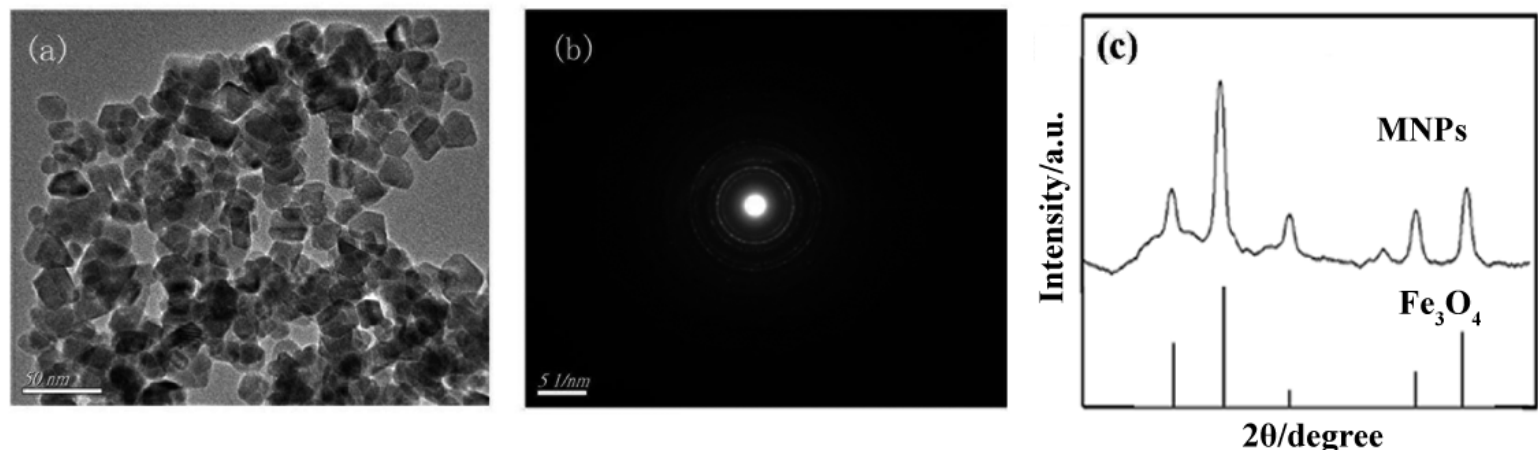

225

Fig. 3. TEM image (a), SAED image (b), and XRD (c) of the amino-functionalized magnetic nanoparticles.

\subsection{Characterization of the antigen modified MNPs and antibody functionalized UCNPs}

In this report, to verify the formation of the bionanoparticles, infrared spectroscopy was utilized to monitor the reaction products in each derivatization step, and the results are shown in Fig. 4: spectra of UCNPs (A), carboxylation-UCNPs (B), carboxylation-UCNPs-antibody probes (C), amination-MNPs (D) and amination-MNPs-antigen. Fig. 4 (A, B, C, D and E) confirmed the presence of carboxyl on the UCNPs, UCNP-antibody probes, UCNP-antibody-antigen-MNPs compounds and antigen on the MNPs. More specifically, in Fig. 4 (A, B, C), the water-soluble UCNPs presented with a single broad peak at $3427 \mathrm{~cm}^{-1}$, corresponding to the stretching vibration of hydroxide radicals $(-\mathrm{OH})$. The characteristic peak at $1629 \mathrm{~cm}^{-1}$ is related to the asymmetric stretching vibration of carboxyl groups (-COOH) of the citrate ligands on the surface of the UCNPs. These two peaks indicated that the carboxyl groups from the ligand exchange were successfully modified on the surface of UCNPs to produce water-soluble UCNPs. When the glutaraldehyde crosslinking method -prepared antibodies were introduced, three characteristic peaks at 2360, 2335, and $1396 \mathrm{~cm}^{-1}$ appeared. The peaks at 2360 and $2335 \mathrm{~cm}^{-1}$ corresponded to methylene stretching vibrations $\left(-\mathrm{CH}_{2}-\right)$. The peak at $1396 \mathrm{~cm}^{-1}$ corresponded to carboxyl stretching vibrations (COO-) 
243 due to the linking reaction between the water-soluble UCNPs and the antibodies. Furthermore, a new 244 peak was observed at $1540 \mathrm{~cm}^{-1}$ upon comparison of the spectra of the 245 UCNP-antibody-antigen-MNPs complex and the UCNP-antibody probe; this peak is attributed to 246 the distinct amide I and amide II vibration modes characteristic of antigen proteins. On the other 247 hand, in Fig. 4 (D, E), a new peak was observed at $1400 \mathrm{~cm}^{-1}$ upon comparison of the spectra of the 248 amino-MNPs complex and the MNPs-antigen probes; this peak is attributed to the distinct amide I and amide II vibration modes characteristic of antigen proteins. In the FT-IR spectra of 250 antigen-functionalized- $\mathrm{Fe}_{3} \mathrm{O}_{4} \mathrm{MNPs}$ and antibody-functionalized-UCNPs, all the characterized peaks 251 of $\mathrm{Fe}_{3} \mathrm{O}_{4}$ MNPs and UCNPs appeared in the corresponding wavenumbers, indicating the 252 modification of antigen and antibody onto the surface of MNPs and UCNPs. On the basis of these 253 characterizations, the proposed UCNP-based method is suitable for sensing toxin.

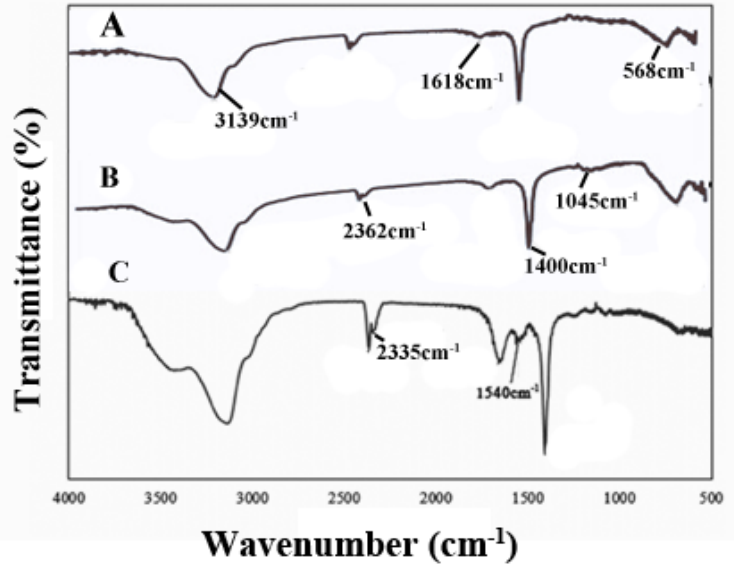

254

255

\subsection{Specific Capturing Evaluation}

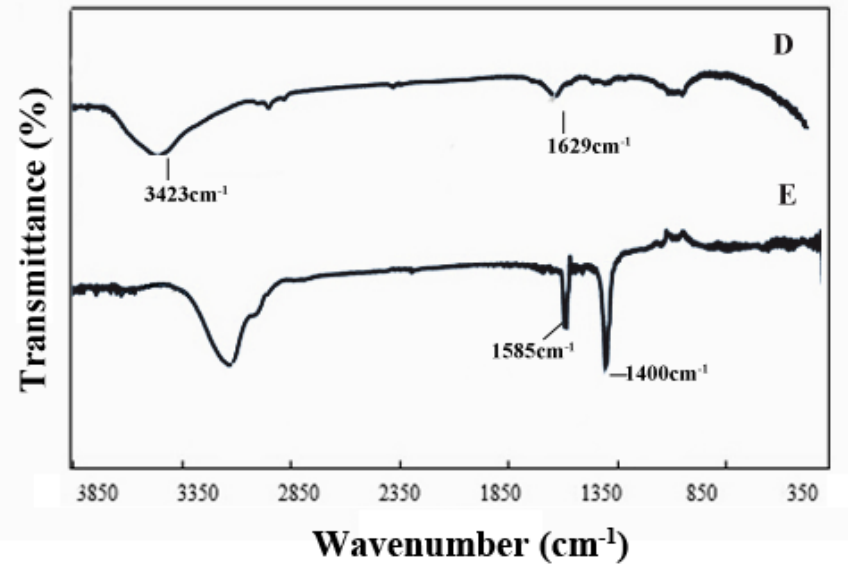

(A), carboxylation-UCNPs

(B),

Fig.4. FT-IR spectrums of oleic acid-capped UCNP

$(\mathrm{A})$$$
\text { carboxylation-UCNPs-antibody probes (C), amination-MNPs (D) and amination-MNPs-antigen (E). }
$$

In order to evaluate the specificity of the immunoassay procedure using this developed fluorescent probe for $A_{F B}$, other two commonly occurring toxins, Aflatoxin $G_{1}\left(A F G_{1}\right)$ and 

were shown in Fig. 5, both $\mathrm{AFG}_{1}$ and $\mathrm{FB}_{1}$ caused negligible changes of the fluorescence, while a significant change of fluorescence was observed for AFB 1 . Therefore, it is clearly demonstrated that 263 the designed fluorescent probe has good specificity to capture $\mathrm{AFB}_{1}$.

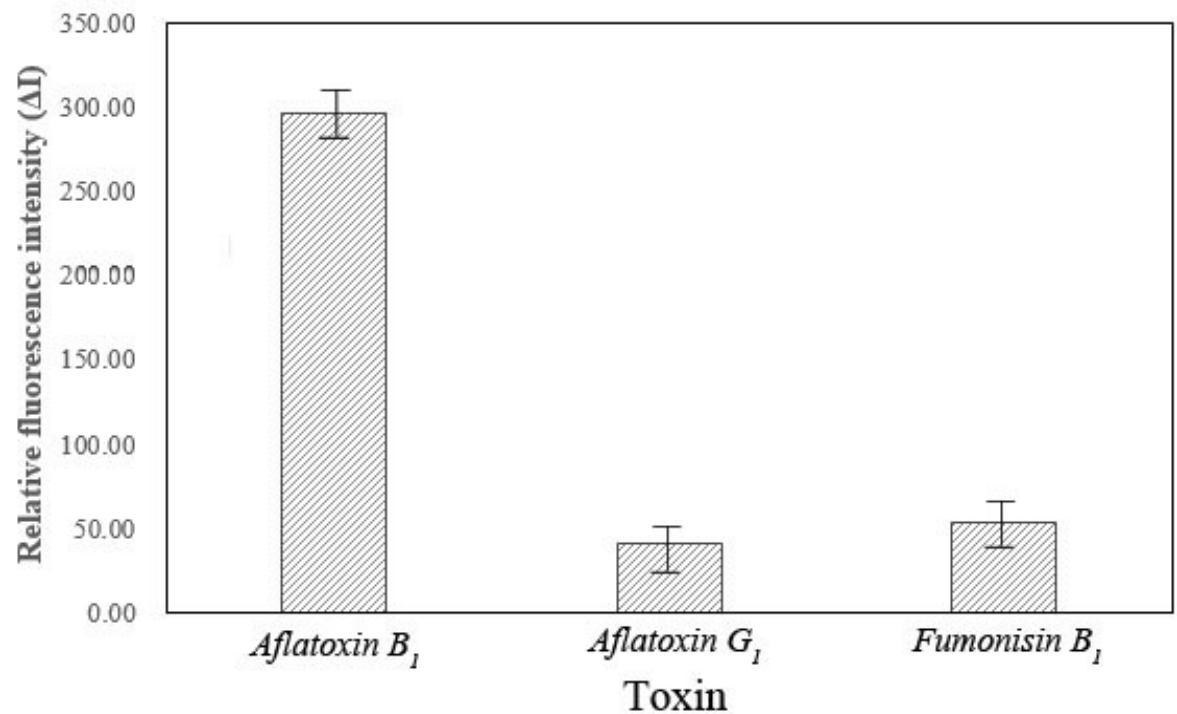

Fig. 5.Specific selectivity evaluation of the proposed method for $\mathrm{AFB}_{1}\left(1 \mathrm{ng} \cdot \mathrm{ml}^{-1}\right)$ against other toxin $\left(1 \mathrm{ng} \cdot \mathrm{ml}^{-1}\right)$.

\subsection{Analytical performance}

In a typical experiment, different concentrations of $\mathrm{AFB}_{1}$ were incubated under agitation with 270 the UCNP-antibody probes for $2 \mathrm{~h}$ at $37{ }^{\circ} \mathrm{C}$. On the basis of the specificity of the antibody for the $271 \mathrm{AFB}_{1}$, UCNPs-antibody-antigen-MNPs complexes were formed. The samples were subsequently 272 concentrated and separated by magnetic for $10 \mathrm{~min}$ to separate the unbound UCNPs-antibody probes. 273 Thereafter, serial dilutions of the supernatants were prepared to examine the fluorescence spectra of 274 the complexes. The $541 \mathrm{~nm}$ emission peak excited by a $980 \mathrm{~nm}$ laser was used to monitor the $\mathrm{AFB}_{1}$ concentration (Lu, Chen, Wang, Zheng, \& Li, 2015). 
277 concentration increased from 0.2 to $100 \mathrm{ng} \cdot \mathrm{mL}^{-1}$. A strong linear correlation $\left(\mathrm{R}^{2}=0.938\right)$ was

278 obtained between various concentrations of $\mathrm{AFB}_{1}(\mathrm{X})$ and the upconversion luminescent intensity 279 (Fig. 6D). In thinner, secondary, and high three separate concentration phases, linear ratios are all 280 higher than 0.90. It can be seen (Fig. 6) that fluorescence intensity has a minimum linear relationship 281 with lowest concentrations $\left(\mathrm{R}^{2}=0.904\right)$, which is due to the UCNPs nano-particles detection 282 precision; fluorescence intensity has a best linear relationship with high concentrations of $\mathrm{AFB}_{1}\left(\mathrm{R}^{2}\right.$ $283=0.9822$ ) because of the dense solution and immunization specific recognition precision. The 284 detection limit of this proposed method for $\mathrm{AFB}_{1}$ was found to be $0.2 \mathrm{ng} \cdot \mathrm{mL}^{-1}$. The precision 285 expressed as the relative standard deviation (RSD) of this detection is 3.56\% (obtained from a series 286 of 10 standard samples each containing $0.4 \mathrm{ng} \cdot \mathrm{mL}^{-1}$ ). Fig. 6 also depicts a typical recording output 287 for the detection of $\mathrm{AFB}_{1}$ with different concentrations. Overall, these results demonstrate that the 288 developed method applied here have a good potential to be used as a rapid screening for the detection of mycotoxin ingrain crops. 

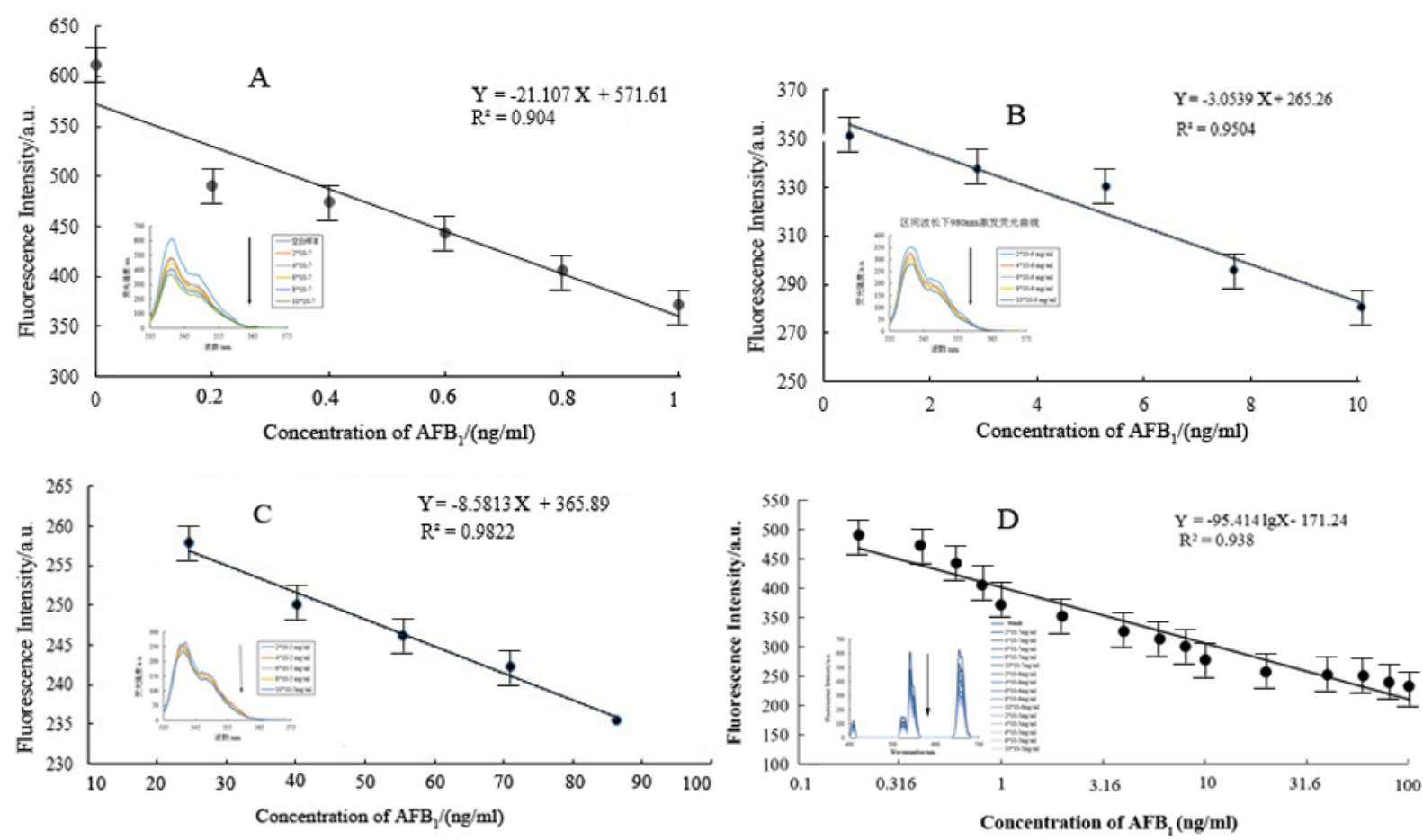

Fig. 6. Linear relation between upconversion luminescent intensity and the various concentrations of $\mathrm{AFB}_{1}$.

Statistical analysis revealed that the detection limit of $\mathrm{AFB}_{1}$ are equal to $0.2 \mathrm{ng} \cdot \mathrm{mL}^{-1}$, as estimated by using $3 \sigma$. These values are desirable for detection $\mathrm{AFB}_{1}$ in various kinds of foods relative to the maximum acceptable standards of these mycotoxins in China and other countries. The RSD of $\mathrm{AFB}_{1}$ detection was equal to $3.56 \%$ indicating that the developed method exhibited good reproducibility. In the absence of $\mathrm{AFB}_{1}$-BSA-MNPs, the fluorescence intensity of $\mathrm{NaYF}_{4}: \mathrm{Yb}, \mathrm{Er}$ was at a maximum, and in the presence of AFB $_{1}$-BSA-MNPs, the antigen binds with antibody-AFB 1 -UCNPs and causes the fluorescent signal of the unreleased UCNPs gradually decreased. It can be understood as that the more MNPs-antigen- antibody-UCNPs was formed, the fewer antibody-UCNPs were remained, and the fluorescence intensity is weaker. also evaluated by determining the recovery of $\mathrm{AFB}_{1}$.by adding a known quantity of standard solution 
to the test solution. As shown in Table 1, the recoveries of $\mathrm{AFB}_{1}$ were between $90.1 \%$ and $113.4 \%$, indicating a high level of accuracy of the developed immunoassay. These analyses demonstrated that

Table 1: Recovery results for $\mathrm{AFB}_{1}$ detection

\begin{tabular}{ccccc}
\hline Samples & $\begin{array}{c}\text { Background } \\
\text { concentration }\left(\mathrm{ng} \cdot \mathrm{ml}^{-1}\right)\end{array}$ & $\begin{array}{c}\text { Added concentration } \\
\left(\mathrm{ng} \cdot \mathrm{ml}^{-1}\right)\end{array}$ & $\begin{array}{c}\text { Detected concentration } \\
\left(\mathrm{ng} \cdot \mathrm{ml} \mathbf{H}^{-1}\right)(\text { mean } \pm \mathrm{SD})\end{array}$ & Recovery radio\% \\
\hline $\mathrm{AFB}_{1}$ & 0.052 & 0.1 & $0.150 \pm 0.032$ & 98 \\
$\mathrm{AFB}_{1}$ & 0.052 & 1 & $0.98 \pm 0.120$ & 92.8 \\
$\mathrm{AFB}_{1}$ & 0.734 & 0.5 & $1.301 \pm 0.233$ & 113.4 \\
$\mathrm{AFB}_{1}$ & 0.734 & 1 & $1.720 \pm 0.121$ & 98.6 \\
$\mathrm{AFB}_{1}$ & 3.364 & 1 & $4.265 \pm 0.236$ & 90.1 \\
$\mathrm{AFB}_{1}$ & 3.364 & 5 & $8.465 \pm 0.103$ & 102.02 \\
\hline
\end{tabular}

\section{Conclusions}

In this study, rare earth doped upconversion nanoparticles have been successfully assembled for sensing Aflatoxins $\mathrm{B}_{1}$ in actual food samples (peanut oil). Herein, antigen-modified magnetic nanoparticles were used for immunosensing probes, and antibody functionalized $\mathrm{NaYF}_{4}$ upconversion nanoparticles as color signal probes. Due to strong fluorescence signal, low autofluorescence of the UCNPs, rapid separation and purification of the magnetic nanoparticles and the immunocomplex, this method can reduce significantly the overall assay time. Based on these

317 results, the ease of use and reliability, the developed method could be extended for the rapid 318 detection of other toxins in the edible oils and other agricultural products. suggest that it maybe be extended to other agriculture products 


\section{Acknowledgments}

323 This work has been financially supported by the National Natural Science Foundation of China 324 (31471646) and a Project Funded by the Priority Academic Program Development of Jiangsu Higher 325 Education Institutions (PAPD).

327 Conflict of interest

328 The authors declare no conflicts of interest. The authors alone are responsible for the content of 329 this manuscript.

330 


\section{References}

Ahn, K.S., Lim, K. R., Jeong, D., Lee, B. Y., Kim, K. S., \& Lee, W.Y. (2016). Fluorescence energy transfer inhibition bioassay for cholera toxin based on galactose-stabilized gold nanoparticles and amine-terminated quantum dots. Microchemical Journal, 124, 9-14.

Aramburu, I., Galban, J., Ostra, M., Ubide, C., Vidal, M., \& Zuriarrain, J. (2015). Uncertainty in CCD detectors with and without cooling devices when used for molecular fluorescence measurements. Analytical Methods, 7(6), 2379-2385.

Boyer, J.C., Manseau, M.P., Murray, J. I., \& van Veggel, F. C. J. M. (2010). Surface Modification of Upconverting NaYF4 Nanoparticles with PEG-Phosphate Ligands for NIR (800 nm) Biolabeling within the Biological Window. Langmuir, 26(2), 1157-1164.

Busman, M., Liu, J., Zhong, H., Bobell, J. R., \& Maragos, C. M. (2014). Determination of the aflatoxin AFB1 from corn by direct analysis in real time-mass spectrometry (DART-MS). Food Additives \& Contaminants: Part A, 31(5), 932-939.

Ceker, S., Agar, G., Alpsoy, L., Nardemir, G., \& Kizil, H. E. (2014). Antagonistic effects of Satureja hortensis essential oil against AFB, on human lymphocytes in vitro. Cytology and Genetics, 48(5), 327-332.

Chatterjee, D. K., Gnanasammandhan, M. K., \& Zhang, Y. (2010). Small Upconverting Fluorescent Nanoparticles for Biomedical Applications. Small, 6(24), 2781-2795.

Chatterjee, D. K., Rufaihah, A. J., \& Zhang, Y. (2008). Upconversion fluorescence imaging of cells and small animals using lanthanide doped nanocrystals. Biomaterials, 29(7), 937-943.

Chen, H.Q., Yuan, F., \& Wang, L. (2013). Simple and sensitive turn-on luminescent detection of biothiols based on energy transfer between green-emitting upconversion nanocrystals and gold nanoparticles. Analytical Methods, 5(11), 2873-2879.

Chen, K., Fang, J., Peng, X., Cui, H., Chen, J., Wang, F.,Zhou, Y. (2014). Effect of selenium supplementation on aflatoxin B1-induced histopathological lesions and apoptosis in bursa of Fabricius in broilers. Food and Chemical Toxicology, 74, 91-97.

Fang, S., Wang, C., Xiang, J., Cheng, L., Song, X., Xu, L.,Liu, Z. (2014). Aptamer-conjugated upconversion nanoprobes assisted by magnetic separation for effective isolation and sensitive detection of circulating tumor cells. Nano Research, 7(9), 1327-1336.

Gao, J., Gu, H., \& Xu, B. (2009). Multifunctional Magnetic Nanoparticles: Design, Synthesis, and Biomedical Applications. Accounts of Chemical Research, 42(8), 1097-1107.

Herzallah, S. M. (2009). Determination of aflatoxins in eggs, milk, meat and meat products using HPLC fluorescent and UV detectors. Food Chemistry, 114(3), 1141-1146.

Huang, L.J., Yu, R.Q., \& Chu, X. (2015). DNA-functionalized upconversion nanoparticles as biosensors for rapid, sensitive, and selective detection of $\mathrm{Hg}^{2+}$ in complex matrices. Analyst, 140(15), 4987-4990.

Karimi, M., Habibi-Rezaei, M., Safari, M., Moosavi-Movahedi, A. A., Sayyah, M., Sadeghi, R., \& Kokini, J. (2014). Immobilization of endo-inulinase on poly-d-lysine coated $\mathrm{CaCO}_{3}$ micro-particles. Food Research International, $66,485-492$.

Klu, Y. A. K., \& Chen, J. (2015). Effect of peanut butter matrices on the fate of probiotics during simulated gastrointestinal passage. LWT - Food Science and Technology, 62(2), 983-988.

Kozlov, I. A., Melnyk, P. C., Stromsborg, K. E., Chee, M. S., Barker, D. L., \& Zhao, C. (2004). Efficient strategies for the conjugation of oligonucleotides to antibodies enabling highly sensitive protein detection. Biopolymers, 73(5), 621-630.

Lu, Z., Chen, X., Wang, Y., Zheng, X., \& Li, C. (2015). Aptamer based fluorescence recovery assay for aflatoxin $B_{1}$ 
using a quencher system composed of quantum dots and graphene oxide. Microchimica Acta, 182(3-4), 571-578.

Luongo, D., Russo, R., Balestrieri, A., Marzocco, S., Bergamo, P., \& Severino, L. (2013). In vitro study of AFB 1 and $\mathrm{AFM}_{1}$ effects on human lymphoblastoid Jurkat T-cell model. Journal of immunotoxicology, 11(4), 353-358.

Ma, Y., Liu, H., Han, Z., Yang, L., \& Liu, J. (2015). Highly-reproducible Raman scattering of NaYF $: \mathrm{Yb}_{2} \mathrm{Er}_{\mathrm{S}} \mathrm{SiO}{ }_{2} @ \mathrm{Ag}$ for methylamphetamine detection under near-infrared laser excitation. Analyst, 140(15), 5268-5275.

Maragos, C. (2009). Fluorescence polarization immunoassay of mycotoxins: a review. Toxins, 1(2), 196-207.

Mnoyan, A. N., Kirakosyan, A. G., Kim, H., Jang, H. S., \& Jeon, D. Y. (2015). Electrostatic Stabilized InP Colloidal Quantum Dots with High Photoluminescence Efficiency. Langmuir, 31(25), 7117-7121.

Ong, L. C., Ang, L. Y., Alonso, S., \& Zhang, Y. (2014). Bacterial imaging with photostable upconversion fluorescent nanoparticles. Biomaterials, 35(9), 2987-2998.

Passone, M. A., Girardi, N. S., \& Etcheverry, M. (2013). Antifungal and antiaflatoxigenic activity by vapor contact of three essential oils, and effects of environmental factors on their efficacy. LWT - Food Science and Technology, 53(2), 434-444.

Quiles, J. M., Manyes, L., Luciano, F., Mañes, J., \& Meca, G. (2015). Influence of the antimicrobial compound allyl isothiocyanate against the Aspergillus parasiticus growth and its aflatoxins production in pizza crust. Food and Chemical Toxicology, 83, 222-228.

Sai, N., Chen, Y., Liu, N., Yu, G., Su, P., Feng, Y., Gao, Z. (2010). A sensitive immunoassay based on direct hapten coated format and biotin-streptavidin system for the detection of chloramphenicol. Talanta, 82(4), 1113-1121.

Sanders Iii, C. T., DeMasie, C. L., Kerr, W. L., Hargrove, J. L., Pegg, R. B., \& Swanson, R. B. (2014). Peanut skins-fortified peanut butters: Effects on consumer acceptability and quality characteristics. LWT - Food Science and Technology, 59(1), 222-228.

Sharma, A., Rawat, K., Solanki, P. R., \& Bohidar, H. B. (2015). Electrochemical response of agar ionogels towards glucose detection. Analytical Methods, 7(14), 5876-5885.

Sozer, N., \& Kokini, J. L. (2014). Use of quantum nanodot crystals as imaging probes for cereal proteins. Food Research International, 57, 142-151.

Tian, J., Bai, J., Peng, Y., Qie, Z., Zhao, Y., Ning, B.,Gao, Z. (2015). A core-shell-structured molecularly imprinted polymer on upconverting nanoparticles for selective and sensitive fluorescence sensing of sulfamethazine. Analyst, 140(15), 5301-5307.

Van de Perre, E., Jacxsens, L., Lachat, C., El Tahan, F., \& De Meulenaer, B. (2015). Impact of maximum levels in European legislation on exposure of mycotoxins in dried products: Case of aflatoxin B1 and ochratoxin A in nuts and dried fruits. Food and Chemical Toxicology, 75, 112-117.

Waliyar, F., Reddy, S., \& Lava-Kumar, P. (2009). Review of immunological methods for the quantification of aflatoxins in peanut and other foods. Journal Information, 36(1), 54-59.

Wang, F., Deng, R., Wang, J., Wang, Q., Han, Y., Zhu, H., Liu, X. (2011). Tuning upconversion through energy migration in core-shell nanoparticles. Nat Mater, 10(12), 968-973.

Wang, F., Han, Y., Lim, C. S., Lu, Y. H., Wang, J., Xu, J., Liu, X. G. (2010). Simultaneous phase and size control of upconversion nanocrystals through lanthanide doping. Nature, 463(7284), 1061-1065.

Wang, L., Li, P., \& Li, Y. (2007). Down- and Up-Conversion Luminescent Nanorods. Advanced Materials, 19(20), 3304-3307.

Wang, L., \& Li, Y. (2006). Na( $\left.\mathrm{Y}_{1.5} \mathrm{Na}_{0.5}\right) \mathrm{F} 6$ Single-Crystal Nanorods as Multicolor Luminescent Materials. Nano Letters, 6(8), 1645-1649.

Wang, Z., Yue, T., Yuan, Y., Cai, R., Niu, C., \& Guo, C. (2013). Preparation of immunomagnetic nanoparticles for the separation and enrichment of Alicyclobacillus spp. in apple juice. Food Research International, 54(1), 302-310. 
Xia, Q., Huang, X.Y., Xue, F., Zhang, J.J., Zhai, B., Kong, D.C., Long, X.D. (2013). Genetic polymorphisms of DNA repair genes and DNA repair capacity related to aflatoxin $\mathrm{b}_{1}\left(\mathrm{AFB}_{1}\right)$-induced DNA damages. New Research Directions in DNA Repair, 1, 377-412.

Xu, X., Liu, X., Li, Y., \& Ying, Y. (2013). A simple and rapid optical biosensor for detection of aflatoxin $\mathrm{B}_{1}$ based on competitive dispersion of gold nanorods. Biosensors and Bioelectronics, 47(0), 361-367.

Yang, B.c., Wang, F., Deng, W., Zou, Y., Liu, F.y., Wan, X.d., Huang, O.p. (2015). Wooden-tip electrospray ionization mass spectrometry for trace analysis of toxic and hazardous compounds in food samples. Analytical Methods, 7(14), 5886-5890.

Zhang, Y., Wu, L., Tang, Y., Su, Y., \& Lv, Y. (2014). An upconversion fluorescence based turn-on probe for detecting lead(ii) ions.. Analytical Methods, 6(22), 9073-9077.

Zhou, J., Liu, Z., \& Li, F. (2012). Upconversion nanophosphors for small-animal imaging. Chemical Society Reviews, 41(3), 1323-1349. 